\section{How we manage smoldering multiple myeloma}

\author{
Alessandra Romano, ${ }^{1 *}$ \\ Claudio Cerchione, ${ }^{2 *}$ \\ Concetta Conticello, ${ }^{3}$ \\ Giovanni Martinelli, ${ }^{2}$ \\ Francesco Di Raimondo ${ }^{1,3}$
}

${ }^{1}$ Dipartimento di Chirurgia e Specialità

Medico-Chirurgiche, Sezione di

Ematologia, Università degli Studi di

Catania; ${ }^{2}$ Hematology Unit, Istituto

Scientifico Romagnolo per lo Studio e la

Cura dei Tumori (IRST) IRCCS,

Meldola (FC); ${ }^{3}$ U.O.C. di Ematologia,

Azienda Policlinico Rodolico San Marco,

Catania, Italy

\begin{abstract}
Smoldering myeloma (SMM) is an asymptomatic stage characterized by bone marrow plasma cells infiltration between 10$60 \%$ in absence of myeloma-defining events and organ damage. Until the revision of criteria of $\mathrm{MM}$ to require treatment, two main prognostic models, not overlapping each other, were proposed and used differently in Europe and in US. Novel manageable drugs, like lenalidomide and monoclonal antibodies, with high efficacy and limited toxicity, improvement in imaging and prognostication, challenge physicians to offer early treatment to highrisk SMM. Taking advantage from the debates offered by SOHO Italy, in this review we will update the evidence and consequent clinical practices in US and Europe to offer readers a uniform view of clinical approach at diagnosis, follow-up and supportive care in the SMM setting.
\end{abstract}

\section{Introduction}

For long time, the two terms of Smoldering and Indolent myeloma were variably used in an undefined manner, 1,2 until 2003 when the International Myeloma Working Group (IMWG) defined SMM as an asymptomatic stage of plasma cell disorder, defined by the presence of a serum monoclonal component of at least $30 \mathrm{~g} / \mathrm{L}$ and/or more than $10 \%$ plasma cells in the bone marrow (BMPC), ${ }^{3}$ higher than those generally seen in monoclonal gammopathy of uncertain significance (MGUS), in absence of myelomadefining events, like hypercalcemia, renal failure, anemia, or bone disease (also collectively known as CRAB symptoms). In 2014, IMWG included BMPC $>60 \%$, elevated immunoglobulinfree light chains (in which the involved light chains are 100 times more numerous than the uninvolved ones), and 2 or more bone focal lesions identified by magnetic resonance imaging (MRI) ${ }^{4}$ as additional myelomadefining events, that address earlier patients to first-line treatment.

SMM accounts for about $15 \%$ of all the patients with newly diagnosed $\mathrm{MM}^{5}$ and it carriers a higher risk of progression to symptomatic MM compared to MGUS. ${ }^{6,7}$

In the first 5 years after diagnosis the risk of progression to MM in SMM is approximately $10 \%$ per year ${ }^{8}$ and decreases thereafter, differently from MGUS in which the rate of progression to $\mathrm{MM}$ is $1 \%$ per year, constant overtime. ${ }^{9}$ The difference in clinical behavior in SMM is due to genetic heterogeneity ${ }^{10}$ as deciphered by application of novel technologies. ${ }^{10-12}$ While transcriptome trajectory is invariant, ${ }^{13}$ genomic events associated to progression from SMM through active MM can follow two main patterns, as revealed by wholegenome sequencing approach. ${ }^{11}$ The first, in which the sub-clonal architecture is retained and the progression is consequence of linear increase of disease burden; the second, due to a change of the sub-clonal architecture, in which progression is associated to stochastic additional complex genomic events. ${ }^{12,14}$ Like in active MM, cytogenetics can identify high-risk SMM patients ${ }^{8}$ and will likely be incorporated in future comprehensive models for risk stratification. ${ }^{15}$ As a whole, BMPC external factors, like microbiote composition $^{16}$ or immune dysfunction, ${ }^{17-21}$ can play a role still to investigate.

\section{Initial diagnostic work-up}

While there are no significant differences between Italy and US in the initial diagnosis work-up, to exclude myelomadefining events, ${ }^{4}$ there are some emerging differences about the way to attribute risk class and further follow-up requirements.

According to 2014 IMWG MM diagnostic criteria ${ }^{4}$ and 2016 ESMO guidelines, ${ }^{22} \mathrm{BM}$ evaluation by aspirate and or biopsy is the standard way to evaluate the number, immune phenotype (to check aberrancies like the absence of CD19 and/or CD45 expression, decreased expression of CD38, and overexpression of CD56) ${ }^{\mathbf{2 3}}$ proliferative index ${ }^{24,25}$ and genetic aberrations (by FISH and/or conventional cytogenetics) of BMPC. Moreover, BM evaluation can provide additional
Correspondence: Alessandra Romano, Dipartimento di Chirurgia e Specialità Medico-Chirurgiche, Sezione di Ematologia, Università degli Studi di Catania, Italy.

E-mail: sandrina.romano@gmail.com

Claudio Cerchione, Hematology Unit, Istituto Scientifico Romagnolo per lo Studio e la Cura dei Tumori (IRST) IRCCS, Via P. Maroncelli 40, 47014, Meldola (FC), Italy.

E-mail: claudio.cerchione@irst.emr.it

Key words: Smoldering multiple myeloma.

*Alessandra Romano and Claudio Cerchione equally contributed as co-first authors.

Conflict of interest: the authors declare no potential conflict of interest.

This work is licensed under a Creative Commons Attribution-NonCommercial 4.0 International License (CC BY-NC 4.0).

${ }^{\circ}$ Copyright: the Author(s), 2020

Licensee PAGEPress, Italy

Hematology Reports 2020; 12(s1):8951

doi:10.4081/hr:2020.8951

information, like the presence of dysplastic hematopoiesis, an emerging prognostic factor for active MM. ${ }^{26} \mathrm{~A}$ BM evaluation should be offered to all patients, even if asymptomatic, with a serum monoclonal component higher than $1.5 \mathrm{~g} / \mathrm{dL}$, based on a large Italian study showing that the probability of detecting a plasma cell infiltration $\geq 10 \%$ in asymptomatic patients with a serum M-protein $\leq 15 \mathrm{~g} / \mathrm{L}$ is $4.7 \%$ for IgG subtype. ${ }^{27}$ The absence of significant tumor burden with an $\mathrm{M}$ protein of $<15 \mathrm{~g} / \mathrm{L}$ and a normal FLC ratio seem to predict an MGUS-like behavior. ${ }^{28}$

In several European centers the evaluation is deferred in asymptomatic patients with apparent IgG MGUS if the serum M- protein is $\leq 15 \mathrm{~g} / \mathrm{L}$ and without end-organ damage, until there is evidence of progression to symptomatic disease. ${ }^{29}$ Accordingly, the European Myeloma Network does not routinely recommend bone marrow evaluation when patients have a serum IgG M-protein $\leq 15 \mathrm{~g} / \mathrm{L}$ or IgA Mprotein $\leq 10 \mathrm{~g} / \mathrm{L}$ without CRAB symptoms. ${ }^{29}$ A recent large retrospective series including patients presenting with low risk MGUS profile and no CRAB signs, confirmed that the risk of missing a diagnosis of SMM and MM by omitting bone marrow aspirate and biopsy was less than $1 \%$. Thus, based on comorbidities, frailty, ${ }^{30}$ age and amount of monoclonal component bone marrow evaluation could be deferred, preferring clinical and laboratory monitoring. ${ }^{24,29}$

After the initial diagnosis of SMM, in our Center we repeat laboratory tests, ${ }^{31}$ including a hemogram, biochemistry tests, serum free 
light chain (sFLC) ratio, serum and urine $\mathrm{s}^{30}$ protein studies in 3 months to confirm the stabilization of the monoclonal component, as well as the absence of anemia, kidney impairment, and hypercalcemia. Shift from negative to positive urine immune-fixation and Bence Jones proteinuria are predictor markers of progression to active MM. ${ }^{30,32,33}$ Dynamic monitoring of $\mathrm{sFLC}^{34}$ and $\mathrm{M}$ component are helpful to identify evolving SMM type, ${ }^{35}$ with an emerging prognostic role. ${ }^{36}$ Based on the pioneering work of Dr. Dispenzieri and colleagues who evaluated disease progression in $273 \mathrm{SMM}$ patients at Mayo Clinics, an involved/uninvolved FLC ratio of $\geq 8$ is a significant risk factor for progression, ${ }^{37}$ and if sFLC ratio rises to $\geq 100$, the 2 year risk of progression approaches $80 \%$, thus to be considered a myelomadefining event in the IMWG guidelines. ${ }^{38}$

\section{Imaging: the emerging role of whole-body MRI}

Imaging is required in the initial workup of any suspected plasma cell disorder, ${ }^{22,30}$ to collect detailed information about the BM involvement, by whole-body $\mathrm{MRI}^{39}$ and to early identify osteolytic bone lesions, preferentially by low-dose whole-body CT. ${ }^{40}$ In $20 \%$ SMM patients the X-rays scans is silent due to bone loss $<30 \%,{ }^{40}$ thus wholebody CT screening for bone lesions can lead to change clinical management in almost one third of patients in the real-life setting. ${ }^{41}$ Latest IMWG guidelines recommended to perform WBCT (either CT alone or as part of an FDG-PET/CT protocol) as the first imaging technique at suspected SMM and, if these images are negative or inconclusive, to perform whole-body MRI. ${ }^{42}$ Indeed, $18 \mathrm{~F}$ fluorodeoxyglucose (FDG) integrated with computed tomography (18F-FDG PET-CT) provides information more valuable than whole body X-rays for the assessment of myeloma bone disease in areas not covered by MRI. ${ }^{43,44}$

Imaging can also provide prognostic additional information. Moulopoulos et al. first demonstrated that in patients with asymptomatic myeloma, time to progression (TTP) was shorter (16 vs 43 months) for patients with abnormal MRI (due to presence of focal, diffuse or variegated pattern) versus normal MRI. ${ }^{45}$ An abnormal marrow signal of MRI of the spine in a patient with SMM was associated with a significant factor for progression to symptomatic myeloma (median 15 months) and confirmed by similar findings in independent cohorts, leading to the new IMWG criteria to identify MM patients. ${ }^{39}$

Since in MM imbalanced distribution of active lesions is frequently observed in medical imaging, associated to spatial heterogeneity, ${ }^{46,47}$ integrative imaging, like whole body MRI or immunoPET, can add valuable information diagnosis and prognosis. The preliminary results of immunoPET scans based on antibody-based radiotracers targeted for $\mathrm{CXCR} 4, \mathrm{BCMA}$ or $\mathrm{CD} 38^{48}$ are shifting imaging from a metabolic toward a functional technique to monitor overtime and in different body areas potential therapeutic targets. ${ }^{44,48}$ The imaging biomarker speed of growth, defined as development of the total tumor volume over time as detected by whole-body MRI, can identify $63.2 \%$ of SMM patients who progress within 2 years, including a high-risk group with a 2-year progression rate of $82.5 \%$. ${ }^{49}$ newly-diagnosed $\mathrm{MM}$ significant splenic signal loss on diffusionweighted MRI (DW-MRI) images, was seen in $24 \%$ patients and preserved in MGUS, reflecting increased tumor burden and associated to inferior outcome ${ }^{50}$, but its role has never been tested in SMM.

\section{Risk assessment}

After the diagnosis of SMM, it is necessary to evaluate the risk of progression to symptomatic disease, integrating several parameters that should be taken in account to predict the risk of progression to symptomatic MM and address potential therapeutic interventions.

The Level and the Type of Serum M Protein Concentration: the size and type of the serum M-protein are two independent significant risk factors for progression in MM. 2,6,51,52 In a large retrospective series, the median time to progression (TTP) in patients with a component $\geq 4 \mathrm{~g} / \mathrm{dL}$ was 18 months vs 75 months in patients with a lower serum M protein; the median TTP was significantly shorter in patients with $\operatorname{IgA}$ versus $\operatorname{IgG} \mathrm{M}$-protein ${ }^{6}$ : however, reclassifying SMM patients according to 2014 IMWG criteria for active MM, size and quality of M-protein have lost their prognostic meaning. ${ }^{8}$ Evolving changes in M-protein and hemoglobin, ${ }^{35,36}$ associated to FLC ratio $\geq 8$, and $\mathrm{BMPC} \geq 20 \%$ clearly identify those patients to requiring restaging with BM biopsy and imaging to validate progression. ${ }^{53}$

Percentage of Bone Marrow Plasma Cells: Based on two large independent series, ${ }^{6,37,54}$ BMPC $>20 \%$ is associated to shorter time progression. This cut-off has been validated in a large series of patients with SMM diagnosed according to 2014 IMWG criteria for active MM and it is part of the $20 / 2 / 20$ IMWG score. ${ }^{8}$

Genomic and transcriptomic abnormalities Genomic aberrancies are associated to increased risk of progression through active MM. ${ }^{14,55-57}$ Among findings available in clinics, the presence of del (17p13), $t(4 ; 14),+1 \mathrm{q} 21$ and hyperdiploidy is associated to inferior TTP. ${ }^{58,59}$ Based on a cohort of 331 patients with MGUS and SMM, Dhodapkar and colleagues identified a gene expression profiling (GEP70-gene signature) signature as an independent predictor of the risk of progression to MM. ${ }^{60}$ The same group identified four genes that can predict high risk of progression from smoldering to symptomatic MM.61 However, these techniques are note reproducible in all centers, require a specific expertise, and are burdened with technical issue such as the necessity to enrich neoplastic plasma cells and to avoid bone marrow hemodilution. Further efforts are required for quality control, harmonization and standardization before wider use in routine practice. ${ }^{62}$

Immunophenotyping: The Spanish group found that $60 \%$ SMM patients have aberrancies in the immunephenotype of BMPCs similar to MM, where $>95 \%$ of PCs are aberrant and only $<5 \%$ of the detected PCs are normal, with a median TTP to symptomatic MM of 34 months. ${ }^{23}$ Similar aberrancies in the phenotype can be monitored also in peripheral blood, looking at circulating Plasma Cells (PBPC). ${ }^{63}$ Patients with high circulating $\mathrm{PBPC}$ have a higher risk to progress to active disease within 2 years compared with patients without high circulating PC (71 versus $25 \%$, respectively). However, the detection of circulating PC is still not standardized and difficult to reproduce, ${ }^{64}$ despite in the last years a terrific effort is challenging data interpretation and prospective clinical trial design of subsequent studies to incorporate and harmonize flow cytometry for ${ }^{65}$ disease assessment in both smoldering and active MM. ${ }^{62,63,66}$

Immunoparesis: the suppression of one or more uninvolved immunoglobulins is a significant risk factor for progression in SMM, as shown by two independent large series. In the Mayo Clinics' experience, the median TTP was 159 months for patients without immunoparesis, 89 months in those with a reduction of only one isotype, and 32 months in patients with reduction in two isotypes of uninvolved immunoglobulins. The Spanish group reported similar findings, with a median TTP of 31 months in SMM patients carrying one or more reduced 
uninvolved immunoglobulins. ${ }^{23}$

Serum-Free Light-Chain Ratio: Based on the first work of the Mayo Clinic group, including 273 SMM patients, an involved/uninvolved FLC ratio of $\geq 8$ is a significant risk factor for progression. ${ }^{37}$ When the involved/uninvolved FLC ratio rises to $\geq 100$, the median TTP is only 15 months, and the 2-year risk of progression approaches $80 \%$. Therefore, this can be considered as a biomarker of early progression and such patients are now classified as MM. ${ }^{38}$ However, recent studies suggest that this cut-off for sFLC may not confer as high a risk as initially defined, ${ }^{67}$ and additional factors should be added, thus conveying that a single biomarker cannot be predictive for evolutionary trajectory in SMM trough progression to symptomatic MM. ${ }^{15}$

Prediction models In lack of a single reliable biomarker, clinical and laboratory findings should be integrated. To this end, several models and relevant scores have been developed and tested in clinical trials. ${ }^{15}$

In US the risk assessment of progression to MM in SMM is largely based on the Mayo Clinic (version 2007, 2008 developed before the 2014 update in the MM criteria ${ }^{4,22}$ and Arkansas models.

The Mayo 2007 score takes in account only two lab findings, BMPC $\geq 10 \%$ and serum $M$ protein $\geq 3 \mathrm{~g} / \mathrm{dL}$ to identify three groups of patients with the risk of progression to active MM at 5 years of $15 \%$, $43 \%$ and $69 \%$ respectively. Adding FLC ratio $>8$, the 5 -year progression rates were $25 \%, 51 \%$, and $76 \%$, in the presence of one, two, or three risk factors respectively, in the Mayo 2008 score. ${ }^{37}$ Taking in account the 2014 update of the MM criteria the score has been further improved in the 20/2/20 Mayo 2018 version, combining the presence of BMPCs $>20 \%$, a value of M-component $>2$ $\mathrm{g} / \mathrm{dL}$ and $\mathrm{sFLC}$ ratio $>20$ to identify three groups of patients with the risk of progression to active $\mathrm{MM}$ at 5 years of $22.5 \%, 46.7 \%$ and $81.5 \%$ respectively. ${ }^{8}$

The Arkansas risk-stratification model is based on gene-expression of 4 genes, M protein $\geq 3 \mathrm{~g} / \mathrm{dL}$ and albumin level $<3 \mathrm{~g} / \mathrm{dL}$ to identify three groups of patients with the risk of progression to active MM at 2 years of $5 \%, 44.8 \%$ and $85.7 \%$ respectively. ${ }^{68}$

In Europe, the Spanish group proposed the PETHEMA score, developed before the 2014 update in the MM criteria ${ }^{4}$ on a cohort of 106 patients, combining the presence of aberrant BMPCs (aPCs/BMPC $\geq 95 \%$ ) and immunoparesis to address the risk of progression to active $\mathrm{MM}$ at 5 years is $4 \%$, $46 \%$, and $72 \%$, for patients with none, 1 , or
2 risk factors respectively. ${ }^{23}$ The Danish group suggested a model derived form a population-based study, involving 297 patients, in which combining $\mathrm{M}$ protein $\geq 3$ $\mathrm{g} / \mathrm{dL}$ an immunoparesis could distinguish three groups of patients with the risk of progression to active MM at 5 years of $9 \%$, $24 \%$ and $55 \%$ respectively. ${ }^{6}$

So far, the Mayo 2008 and the PETHEMA models have been used and validated in prospective trials. However, the two models do not overlap and there are many patients that are differently classified according the two models, ${ }^{70}$ thus most investigators use the 20/2/20 Mayo 2018 score based on parameters (M-protein size and the amount of BMPCs) available and reproducible in all centers.

\section{Follow up}

Outside the clinical trial setting, additional examinations should be recommended only in case of clinical evidence for progressive disease from the routine work-up. Subsequent follow-up should be individualized, based on risk of progression, evaluated by application of one of the above-mentioned scores and life expectancy.

The EMN and IMWG recommends follow-up 3 months after the initial SMM diagnosis, and if the results are stable, follow-up should be every 4-6 months for a year, and then every 6-12 months.

Since the likelihood of finding bone lesions at skeletal survey is very low for Mprotein $\operatorname{IgG} \leq 15 \mathrm{~g} / \mathrm{L}(2 \%)$ and $\operatorname{IgA} \leq 10 \mathrm{~g} / \mathrm{L}$ $(0.0 \%),{ }^{27}$ in Europe imaging evaluation is not routinely recommended when patients have a serum $\operatorname{IgG}$ M-protein $\leq 15 \mathrm{~g} / \mathrm{L}$ or IgA M-protein $\leq 10 \mathrm{~g} / \mathrm{L}$ without bone pain, ${ }^{29}$ and for asymptomatic patients with limited life expectancy. $^{29}$ In US, based on IMWG recommendations, imaging MRI is performed on an annual basis for at least 5 years and later based on clinical suspicion of progression, according to the findings of a large study at Mayo Clinics that suggested new cut-offs for prognostic variables to risk stratify SMM patients, the Mayo score $20 / 2 / 20$, showing a stabilization of risk progression at $3 \%-5 \%$ per year beyond 5 years of follow-up. ${ }^{8}$

In our Center, we generally use the 20/2/20 Mayo 2018 prognostic scoring system, ${ }^{8}$ associated to CT-scan findings, evolving nature of the M-protein and sFLC, and Bence Jones proteinuria and distinguish SMM patients for further the follow-up in three categories:

- low risk, with a probability of progression at 5 years less than $10 \%$, that should be followed similarly to MGUS patients, every 6 months in the first two years and then every 12 months

- intermediate risk with a probability of progression at 5 years less than $50 \%$. They represent the true SMM patients that should be followed every 3-6 months

- high risk, with a probability of progression at 2 years more than $50 \%$, for whom is under investigation the need of early treatment. Based on current evidences, outside of the clinical trial setting, treatment for SMM or MGUS is not recommended and treatment should be given only in case of symptomatic progression, as detected by the presence of one of the myeloma-defining events. ${ }^{4,22}$

In our Center, we propose at baseline CT-scan, bone marrow biopsy and aspirate to asymptomatic patients when Mcomponent in serum is higher than $1 \mathrm{~g} / \mathrm{dL}$ for IgA subtype and $15 \mathrm{~g} / \mathrm{L}$ for IgG subtype, ${ }^{27,29}$ and to all patients with Bence Jones proteinuria due to the increased probability to progress to high-risk SMM and active MM. ${ }^{32,33,71}$ Further imaging by CT-scan or MRI is performed every $18-24$ months in lack of bone pain or if not differently clinically indicated.

The dynamic evaluation of additional lab findings can be helpful, including the increase of at least $25 \%$ of M-protein or sFLC over time and hemoglobin reduction by at least $0.5 \mathrm{~g} / \mathrm{dL}$ within the first year, that prompt us to reduce the follow up lag time to every 2 months, to detect the presence of one of the myeloma-defining events which require active anti-MM treatment. If after the second year of follow up M-protein, s-FLC and hemoglobin remain stable, the followup schedule can change to one visit per year. In addition, at least once a year we evaluate Pro-BNP and total urine protein to detect any cardiac or renal impairment that could lead to a diagnosis of amyloidosis.

\section{Clinical management and treat- ment}

Currently, management of SMM, especially of high-risk patients, is challenging, also because the available risk stratification models do not help to predict accurately the risk of progression. In the uncertainty to overtreat asymptomatic patients without improving quality of life or overall survival, waiting for mature results of completed or ongoing clinical trials (see below), no drugs are approved for the 
treatment. For each individual patient a close observation remains the standard of care, taking in account the requirements of supportive and preventive measures to reduce the incidence of impaired bone mineral density, recurrent infections and cardiovascular disease and to optimize timing to start treatment when $\mathrm{MM}$ is diagnosed $^{72}$, even if the contribution of previous diagnosis of asymptomatic disease has been formally shown only for MGUS patients, ${ }^{73,74}$ and this approach reflects the importance of monitoring tumor load in a linear evolution from asymptomatic through active MM. ${ }^{6-8}$

\section{Supportive care}

In MGUS and SMM patients there is an increased incidence of reduced bone mineral density, ${ }^{75}$ osteoporosis and atraumatic fractures, associate to lower levels of vitamin D. ${ }^{76}$ In these cases, beyond a careful evaluation of myeloma defining event by imaging as discussed above, supportive care should include monitoring and supplementation of vitamin D and calcium could be helpful, despite data from large prospective cohorts miss. ${ }^{76}$ In the past, early intervention with zoledronic acid did not show any advantage in increase overall survival, ${ }^{77}$ but they could still be used to prevent myeloma-related skeleton events.

Clinical implication of immunoparesis is the secondary antibody deficiency, whose biological contribution to MM evolution is still under investigation ${ }^{78}$. As consequence of both innate and cellular immunity, ${ }^{18-}$ 20,26,79-81 immunosenescence, ${ }^{82}$ T-cell anergy and addiction of neoplastic plasma cells to TLR4 signaling ${ }^{83,84}$, SMM patients have increased risk to develop bacterial and viral infections. ${ }^{69,85}$ In patients with active MM, both 13-valent pneumococcal conjugate vaccine (PCV13) and 23-valent pneumococcal polysaccharide vaccine (PPSV23) can increase anti-pneumococcal antibodies, and preliminary observations suggest that vaccination is negatively correlated with disease progression. ${ }^{78,86}$ Even if these preliminary observations have not maturely shown in the SMM setting, it is reasonable that a policy to improve the prevention of viral and bacterial infection should be tested in each single center and offered to all SMM patients with a positive anamnesis for recurrent infections, including immunoglobulin replacement, as suggested by the Canadian group. ${ }^{87}$ We suggest that clinical evaluation and anamnestic aspects are fundamental to decide if may be helpful to prescribe or not substitutive therapy in MM. In our Center, we recommend seasonal flu vaccination and anti-pneumococcal vaccination, with either PCV13 or PPSV23 and offer sub-cutaneous, home delivered, immunoglobulin replacement when more than 3 infective episodes are registered in the previous 6 months, ${ }^{88}$ based on promising results of this approach in MM patients. ${ }^{89}$

Since potentially clonal and dysplastic hematopoiesis may co-exist with MGUS and $\mathrm{MM},{ }^{26,90}$ associated with increased risk of atherosclerotic arterial disease, there is an emerging interest in defining the cardiovascular risk due to M-component. ${ }^{91-}$ ${ }^{93}$ Despite data are not available for SMM, a retrospective series found an increase of cardiovascular events (coronary, peripheral and cerebrovascular) in both MGUS and MM patients, ${ }^{75}$ suggesting that the same could happen in SMM setting. In lack of any trial-derived evidence of specific prophylactic strategy to adopt, cooperative studies involving US and European Institutions of well-identified and classified SMM patients could clarify in a near future how to profile and manage cardiovascular risk in SMM.

\section{Treatment options based on out- come goal: delay progression or curative attempt?}

When ESMO guidelines have been released in 2016 only 15\% MM patients could be cured and for this reason immediate treatment for patients with indolent myeloma could not be recommended, strongly encouraging enrollment in clinical trials for high-risk SMM. ${ }^{4}$ After failing of thalidomide in preventing progression through MM..$^{94-97}$ in the last five years the scenario has been changed and more than 50 trials are ongoing to test the feasibility, safety and efficacy of drugs active in $\mathrm{MM}$ that alone or in combination could be offered to high-risk SMM. Both European and US groups have shown that the early addition of lenalidomide to treatment significantly prevents SMM progression to active MM. ${ }^{98}$

However, there are still major concerns about study design, in particular primary endpoint (time to progression versus overall survival) and inclusion criteria, since the first trials included, in a variable proportion, patients who are classified as MM according to 2014 IMWG criteria, using uniform stratification risk models, to make the results comparable each other and the urgent need of additional surrogate endpoints, like achievement of minimal residual negativity. ${ }^{62}$

There are two main strategies arising from the study designs: delay MM onset or eradicate MM cells in the attempt to offer a cure. According to the first goal, a gentle approach, fixed-term and steroid-free, has been proposed by US investigators; on the opposite, a most aggressive approach, including a total therapy, with induction, autologous stem cell transplantation (ASCT) and maintenance regimen with a curative intention is under investigation. ${ }^{72,99}$

With the main goal to find a cure for MM, investigators from both the Spanish Myeloma Group in Europe and the Mayo Clinics in US, have shown that the early addition of lenalidomide to treatment significantly prevents SMM progression to active MM.

In the Spanish randomized phase 3 study early intervention consisting of nine cycles of lenalidomide-dexamethasone induction, followed by lenalidomide maintenance, compared with observation only in patients with high-risk SMM, defined according to the PETHEMA score, showed longer TTP and OS for the lenalidomide-dexamethasone group (median TTP: not reached vs. 21 months; 3-year OS: $94 \%$ vs. $80 \%$ ). ${ }^{98}$ The PETHEMA trial demonstrated for the first time that early treatment with lenalidomide and dexamethasone delayed time to progression to active disease, and provided a significant improvement in overall survival, ${ }^{99}$ confirmed by the updated follow up at ten years presented at EHA 2020 (Abstract \#EP950). However, major concerns raised from the inclusion criteria, based on the PETHEMA score that are not reproducible in all centers. In addition, in this study the bone lesions were evaluated by $\mathrm{X}$-rays skeleton survey, reflecting the standard practice at that time, and that is probably one of the reasons of the exceptionally short median TTP of 24 months in the arm control. The ancillary substudy of the QUIREDEX trial support the importance of lenalidomide to delay the progression to $\mathrm{MM}$ due to recovery of $\mathrm{T}$ cells activation and proliferation. ${ }^{100}$

To better define the contribution of lenalidomide exposure to delay MM onset in SMM patients, reducing the bias associated to steroids, the E3A06 trial investigated continuous exposure to lenalidomide $25 \mathrm{mg}$ versus observation, achieving in the experimental arm 91\% 3-yrs PFS, confirming the promising role of lenalidomide in delay onset of MM-defining events or end-organ damage but confirming that the benefit of lenalidomide treatment is limited to high-risk SMM patients. ${ }^{101}$

Other pilot trials are investigating the role of immunotherapy using monoclonal antibodies as single agents, including elotuzumab (anti-SLAMF7) tested in a phase II study, ${ }^{102}$ daratumumab (anti-CD38) tested in a phase II study, ${ }^{103}$ siltuximab (anti-IL6) ${ }^{104}$ and pembrolizumab (anti PD-1) $)^{105}$ 
extensively described in an excellent recent review, ${ }^{106}$ we recommend our readers for further details.

Additional single agents that more directly engage the immune system, tested in phase I-II studies, include pan-KIR2D inhibitor IPH2101, 107 the anti-human antiintercellular adhesion molecule-1 monoclonal antibody BI-505, ${ }^{108}$ rice bran arabinoxylan and curcumin. ${ }^{109,110}$

The GEM-CESAR phase II single-arm clinical trial led by Spanish Myeloma Group enrolled 90 high-risk SMM (defined according to PETHEMA score) patients who received induction with six 4-week cycles of carfilzomib, lenalidomide and dexamethasone (KRd) regimen, followed by single ASCT, KRd consolidation and maintenance with lenalidomide. Preliminary results from 77 patients who completed induction, HDT-ASCT, consolidation, and 1 $\mathrm{yr}$ of maintenance, showed that $81 \%$ of patients achieved $\geq C R$ and $62 \%$ were MRD negative (Mateos. ASH 2019. Abstr 781).

The safety and efficacy of KRd regimen have been confirmed in a small cohort of high-risk SMM treated at MSKCC in New York, with more $90 \%$ of MRD-negative responses, but longer follow-up is required for definitive conclusions. ${ }^{111}$

There are still open questions before to prime treatment to all SMM patients:

1. What characteristics of immune status, singularly or in cooperation, and mutational signatures co-vary with racial/ethnic differences for asymptomatic MM onset and how these factors influence progression to active MM?

2. Are there differences in immunological triggers (e.g. microbiote composition, dietary and life style) that could modify the evolution pattern and response to treatment?

\section{Conclusions: unmet clinical needs and open questions}

In conclusion, to manage each newly diagnosed SMM patient, it is necessary to identify the risk of progression to individualize follow-up schedule, taking in account all the available data, in a dynamic perspective. Waiting for the results of ongoing clinical trials enrolling SMM patients defined according to the 20/2/20 score, the primary goal of clinical management is delaying onset of CRAB symptoms and improving quality of life. Real-world life experiences will be needed in a near future, to explore the impact of advanced age, co-morbidities and the possibility to reduce drug dosage and exposure.

If early treatment could cure SMM patients, conveying sustained MRD negativity and longer overall survival, without giving unreasonable adverse events and secondary neoplasms, is a challenging paradigm of near future.

\section{References}

1. Kyle RA, Greipp PR. Smoldering multiple myeloma. The New England journal of medicine. 1980;302(24):1347-1349.

2. Alexanian R. Localized and indolent myeloma. Blood. 1980;56(3):521-525.

3. Kyle R, Gertz M, Witzig T, et al. Review of 1027 patients with newly diagnosed multiple myeloma. Mayo Clin Proc. 2003;78(1):21 - 33.

4. Rajkumar SV, Dimopoulos MA, Palumbo A, et al. International Myeloma Working Group updated criteria for the diagnosis of multiple myeloma. Lancet Oncol. 2014;15(12):e538-548

5. Kristinsson SY, Holmberg E, Blimark C. Treatment for high-risk smoldering myeloma. The New England journal of medicine. 2013;369(18):1762-1763.

6. Kyle RA, Remstein ED, Therneau TM, et al. Clinical course and prognosis of smoldering (asymptomatic) multiple myeloma. N Engl J Med. 2007;356(25): 2582-2590.

7. Kyle RA, Therneau TM, Rajkumar SV, et al. A long-term study of prognosis in monoclonal gammopathy of undetermined significance. N Engl J Med. 2002;346(8):564-569.

8. Lakshman A, Rajkumar SV, Buadi FK, et al. Risk stratification of smoldering multiple myeloma incorporating revised IMWG diagnostic criteria. Blood Cancer J. 2018;8(6):59.

9. Rajkumar SV, Landgren O, Mateos MV. Smoldering multiple myeloma. Blood. 2015;125(20):3069-3075.

10. Dutta AK, Fink JL, Grady JP, et al. Subclonal evolution in disease progression from MGUS/SMM to multiple myeloma is characterised by clonal stability. Leukemia. 2019;33(2):457-468.

11. Bolli N, Maura F, Minvielle S, et al. Genomic patterns of progression in smoldering multiple myeloma. Nature Communications. 2018;9(1):3363.

12. Maura F, Petljak M, Lionetti M, et al. Biological and prognostic impact of APOBEC-induced mutations in the spectrum of plasma cell dyscrasias and multiple myeloma cell lines. Leukemia. 2018;32(4):1044-1048.

13. Storti P, Agnelli L, Palma BD, et al. The transcriptomic profile of CD138(+) cells from patients with early progression from smoldering to active multiple myeloma remains substantially unchanged. 2019;104(10):e465-e469.

14. Bustoros M, Sklavenitis-Pistofidis R, Park J, et al. Genomic Profiling of Smoldering Multiple Myeloma Identifies Patients at a High Risk of Disease Progression. Journal of Clinical Oncology. 2020;38(21):2380-2389.

15. Kunacheewa C, Manasanch EE. Highrisk smoldering myeloma versus early detection of multiple myeloma: Current models, goals of therapy, and clinical implications. Best Pract Res Clin Haematol. 2020;33(1):101152.

16. Calcinotto A, Brevi A, Chesi M, et al. Microbiota-driven interleukin-17producing cells and eosinophils synergize to accelerate multiple myeloma progression. Nature Communications. 2018;9(1):4832.

17. Zavidij O, Haradhvala NJ, Mouhieddine $\mathrm{TH}$, et al. Single-cell RNA sequencing reveals compromised immune microenvironment in precursor stages of multiple myeloma. Nature Cancer. 2020;1(5):493-506.

18. Perez C, Botta C, Zabaleta A, et al. Immunogenomic identification and characterization of granulocytic myeloid-derived suppressor cells in multiple myeloma. Blood. 2020;136(2):199-209.

19. Romano A, Parrinello NL, Simeon V, et al. High-density neutrophils in MGUS and multiple myeloma are dysfunctional and immune-suppressive due to increased STAT3 downstream signaling. Scientific Reports. 2020;10 (1):1983

20. Puglisi F, Parrinello NL, Giallongo C, et al. Plasticity of High-Density Neutrophils in Multiple Myeloma is Associated with Increased Autophagy Via STAT3. Int J Mol Sci. 2019;20(14).

21. Barberi C, De Pasquale C, Allegra A, et al. Myeloma cells induce the accumulation of activated CD94low NK cells by cell-to-cell contacts involving CD56 molecules. Blood Adv. 2020;4(10):2297-2307.

22. Moreau P, San Miguel J, Sonneveld P, et al. Multiple myeloma: ESMO Clinical Practice Guidelines for diagnosis, treatment and follow-up $\dagger$. Annals of Oncology. 2017;28:iv52iv61. 
23. Perez-Persona E, Vidriales MB, Mateo $\mathrm{G}$, et al. New criteria to identify risk of progression in monoclonal gammopathy of uncertain significance and smoldering multiple myeloma based on multiparameter flow cytometry analysis of bone marrow plasma cells. Blood. 2007;110(7):25862592.

24. Sidiqi MH, Aljama M, Kumar SK, et al. The role of bone marrow biopsy in patients with plasma cell disorders: should all patients with a monoclonal protein be biopsied? Blood Cancer Journal. 2020;10(5):52.

25. Aljama MA, Sidiqi MH, Lakshman A, et al. Plasma cell proliferative index is an independent predictor of progression in smoldering multiple myeloma. Blood Adv. 2018;2(22):3149-3154.

26. Maia C, Puig N, Cedena MT, et al. Biological and clinical significance of dysplastic hematopoiesis in patients with newly diagnosed multiple myeloma. Blood. 2020;135(26):23752387.

27. Mangiacavalli S, Cocito F, Pochintesta L, et al. Monoclonal gammopathy of undetermined significance: a new proposal of workup. Eur J Haematol. 2013;91(4):356-360.

28. Bustoros M, Kastritis E, SklavenitisPistofidis R, et al. Bone marrow biopsy in low-risk monoclonal gammopathy of undetermined significance reveals a novel smoldering multiple myeloma risk group. Am J Hematol. 2019;94(5):E146-e149.

29. van de Donk NW, Mutis T, Poddighe PJ, Lokhorst HM, Zweegman S. Diagnosis, risk stratification and management of monoclonal gammopathy of undetermined significance and smoldering multiple myeloma. Int J Lab Hematol. 2016;38 Suppl 1:110-122.

30. González-Calle V, Mateos MV. Monoclonal gammopathies of unknown significance and smoldering myeloma: Assessment and management of the elderly patients. European Journal of Internal Medicine. 2018;58:57-63.

31. Evliyaoglu O, van Helden J, Jaruschewski S, Imöhl M, Weiskirchen R. Reference change values of Mprotein, free light chain and immunoglobulins in monoclonal gammopathy. Clin Biochem. 2019;74:42-46.

32. Cesana C, Klersy C, Barbarano L, et al. Prognostic factors for malignant transformation in monoclonal gammopathy of undetermined significance and smoldering multiple myeloma. J Clin Oncol. 2002;20(6):1625-1634.

33. Weber DM, Dimopoulos MA, Moulopoulos LA, Delasalle KB, Smith T, Alexanian R. Prognostic features of asymptomatic multiple myeloma. Br J Haematol. 1997;97(4):810-814.

34. Markovic U, Leotta V, Tibullo D, et al. Serum free light chains and multiple myeloma: Is it time to extend their application? Clin Case Rep. 2020;8(4):617-624.

35. Rosiñol L, Bladé J, Esteve J, et al. Smoldering multiple myeloma: natural history and recognition of an evolving type. Br J Haematol. 2003;123(4):631636.

36. Fernández de Larrea C, Isola I, Pereira A, et al. Evolving M-protein pattern in patients with smoldering multiple myeloma: impact on early progression. Leukemia. 2018;32(6):1427-1434.

37. Dispenzieri A, Kyle RA, Katzmann JA, et al. Immunoglobulin free light chain ratio is an independent risk factor for progression of smoldering (asymptomatic) multiple myeloma. Blood. 2008;111(2):785-789.

38. Larsen JT, Kumar SK, Dispenzieri A, Kyle RA, Katzmann JA, Rajkumar SV. Serum free light chain ratio as a biomarker for high-risk smoldering multiple myeloma. Leukemia. 2013;27(4):941-946.

39. Hillengass J, Fechtner K, Weber MA, et al. Prognostic significance of focal lesions in whole-body magnetic resonance imaging in patients with asymptomatic multiple myeloma. J Clin Oncol. 2010;28(9):1606-1610.

40. Hillengass J, Moulopoulos LA, Delorme S, et al. Whole-body computed tomography versus conventional skeletal survey in patients with multiple myeloma: a study of the International Myeloma Working Group. Blood Cancer Journal. 2017;7(8):e599. e599.

41. Simeone FJ, Harvey JP, Yee AJ, et al. Value of low-dose whole-body CT in the management of patients with multiple myeloma and precursor states. Skeletal Radiol. 2019;48(5):773-779.

42. Jamet B, Bailly C, Carlier T, et al. Imaging of Monoclonal Gammapathy of Undetermined Significance and Smoldering Multiple Myeloma. Cancers (Basel). 2020;12(2).

43. Zamagni E, Nanni C, Patriarca F, et al. A prospective comparison of $18 \mathrm{~F}$ fluorodeoxyglucose positron emission tomography-computed tomography, magnetic resonance imaging and whole-body planar radiographs in the assessment of bone disease in newly diagnosed multiple myeloma. Haematologica. 2007;92(1):50-55.

44. Ulaner GA, Landgren CO. Current and potential applications of positron emission tomography for multiple myeloma and plasma cell disorders. Best Practice \& Research Clinical Haematology. 2020;33(1):101148.

45. Moulopoulos LA, Dimopoulos MA, Smith TL, et al. Prognostic significance of magnetic resonance imaging in patients with asymptomatic multiple myeloma. Journal of clinical oncology : official journal of the American Society of Clinical Oncology. 1995;13(1):251-256.

46. Rasche L, Chavan SS, Stephens OW, et al. Spatial genomic heterogeneity in multiple myeloma revealed by multiregion sequencing. Nature Communications. 2017;8(1):268.

47. Rasche L, Kortüm KM, Raab MS, Weinhold N. The Impact of Tumor Heterogeneity on Diagnostics and Novel Therapeutic Strategies in Multiple Myeloma. Int J Mol Sci. 2019;20(5).

48. Ulaner GA, Sobol NB, O'Donoghue JA, et al. CD38-targeted Immuno-PET of Multiple Myeloma: From Xenograft Models to First-in-Human Imaging. Radiology. 2020;295(3):606-615.

49. Wennmann M, Kintzelé L, Piraud M, et al. Volumetry based biomarker speed of growth: Quantifying the change of total tumor volume in whole-body magnetic resonance imaging over time improves risk stratification of smoldering multiple myeloma patients. Oncotarget. 2018;9(38).

50. Rasche L, Kumar M, Gershner G, et al. Lack of Spleen Signal on Diffusion Weighted MRI is associated with High Tumor Burden and Poor Prognosis in Multiple Myeloma: A Link to Extramedullary Hematopoiesis? Theranostics. 2019;9(16):4756-4763.

51. Alexanian R, Barlogie B, Dixon D. Prognosis of asymptomatic multiple myeloma. Arch Intern Med. 1988;148(9):1963-1965.

52. Dimopoulos MA, Moulopoulos A, Smith T, Delasalle KB, Alexanian R. Risk of disease progression in asymptomatic multiple myeloma. Am J Med. 1993;94(1):57-61.

53. Atrash S, Robinson M, Slaughter D, et al. Evolving changes in M-protein and hemoglobin as predictors for progression of smoldering multiple myeloma. Blood Cancer Journal. 2018;8(11):107.

54. Kastritis E, Terpos E, Moulopoulos L, 
et al. Extensive bone marrow infiltration and abnormal free light chain ratio identifies patients with asymptomatic myeloma at high risk for progression to symptomatic disease. Leukemia. 2013;27(4):947-953.

55. Bolli N, Maura F, Minvielle S, et al. Genomic patterns of progression in smoldering multiple myeloma. Nat Commun. 2018;9(1):3363.

56. López-Corral L, Mateos MV, Corchete LA, et al. Genomic analysis of high-risk smoldering multiple myeloma. Haematologica. 2012;97(9):1439-1443.

57. Mailankody S, Kazandjian D, Korde N, et al. Baseline mutational patterns and sustained MRD negativity in patients with high-risk smoldering myeloma. Blood Adv. 2017;1(22):1911-1918.

58. Neben K, Jauch A, Hielscher T, et al. Progression in smoldering myeloma is independently determined by the chromosomal abnormalities del(17p), $\mathrm{t}(4 ; 14)$, gain 1q, hyperdiploidy, and tumor load. Journal of clinical oncology : official journal of the American Society of Clinical Oncology. 2013;31(34):4325-4332.

59. Rajkumar SV, Gupta V, Fonseca R, et al. Impact of primary molecular cytogenetic abnormalities and risk of progression in smoldering multiple myeloma. Leukemia. 2013;27(8):17381744.

60. Dhodapkar MV, Sexton R, Waheed S, et al. Clinical, genomic, and imaging predictors of myeloma progression from asymptomatic monoclonal gammopathies (SWOG S0120). Blood. 2014;123(1):78-85.

61. Khan R, Dhodapkar M, Rosenthal A, et al. Four genes predict high risk of progression from smoldering to symptomatic multiple myeloma (SWOG S0120). Haematologica. 2015.

62. Romano A, Palumbo GA, Parrinello NL, Conticello C, Martello M, Terragna C. Minimal Residual Disease Assessment Within the Bone Marrow of Multiple Myeloma: A Review of Caveats, Clinical Significance and Future Perspectives. Front Oncol. 2019;9:699.

63. Sanoja-Flores L, Flores-Montero J, Garcés JJ, et al. Next generation flow for minimally-invasive blood characterization of MGUS and multiple myeloma at diagnosis based on circulating tumor plasma cells (CTPC). Blood Cancer J. 2018;8(12):117.

64. Bianchi G, Kyle RA, Larson DR, et al. High levels of peripheral blood circulating plasma cells as a specific risk factor for progression of smoldering multiple myeloma. Leukemia. 2013;27(3):680-685.

65. Gonsalves WI, Buadi FK, Ailawadhi S, et al. Utilization of hematopoietic stem cell transplantation for the treatment of multiple myeloma: a Mayo Stratification of Myeloma and RiskAdapted Therapy (mSMART) consensus statement. Bone Marrow Transplant. 2019;54(3):353-367.

66. Costa LJ, Derman BA, Bal S, et al. International harmonization in performing and reporting minimal residual disease assessment in multiple myeloma trials. Leukemia. 2020.

67. Wu V, Moshier E, Leng S, et al. Risk stratification of smoldering multiple myeloma: predictive value of free light chains and group-based trajectory modeling. Blood Adv. 2018;2(12):1470-1479.

68. Khan R, Dhodapkar M, Rosenthal A, et al. Four genes predict high risk of progression from smoldering to symptomatic multiple myeloma (SWOG S0120). Haematologica. 2015;100(9):1214-1221.

69. Sørrig R, Klausen TW, Salomo M, et al. Smoldering multiple myeloma risk factors for progression: a Danish population-based cohort study. Eur J Haematol. 2016;97(3):303-309.

70. Mateos MV, San Miguel J. V. Smoldering multiple myeloma. Hematological oncology. 2015;33 Suppl 1:33-37.

71. González-Calle V, Dávila J, Escalante $\mathrm{F}$, et al. Bence Jones proteinuria in smoldering multiple myeloma as a predictor marker of progression to symptomatic multiple myeloma. Leukemia. 2016;30(10):2026-2031.

72. Mateos MV, González-Calle V. Timing of treatment of smoldering myeloma: early treatment. Blood Adv. 2018;2(21):3045-3049.

73. Sigurdardottir EE, Turesson I, Lund $\mathrm{SH}$, et al. The Role of Diagnosis and Clinical Follow-up of Monoclonal Gammopathy of Undetermined Significance on Survival in Multiple Myeloma. JAMA Oncol. 2015;1(2):168-174.

74. Bianchi G, Kyle RA, Colby CL, et al. Impact of optimal follow-up of monoclonal gammopathy of undetermined significance on early diagnosis and prevention of myelomarelated complications. Blood. 2010;116(12):2019-2025; quiz 2197.

75. Kristinsson SY, Pfeiffer RM, Björkholm M, et al. Arterial and venous thrombosis in monoclonal gammopathy of undetermined significance and multiple myeloma: a population-based study. Blood. 2010;115(24):4991-4998.

76. Lipe B, Kambhampati S, Veldhuizen PV, Yacoub A, Aljitawi O, Mikhael J. Correlation between markers of bone metabolism and vitamin D levels in patients with monoclonal gammopathy of undetermined significance (MGUS). Blood Cancer Journal. 2017;7(12):646.

77. Witzig TE, Laumann KM, Lacy MQ, et al. A phase III randomized trial of thalidomide plus zoledronic acid versus zoledronic acid alone in patients with asymptomatic multiple myeloma. Leukemia. 2013;27(1):220-225.

78. Lomas OC, Mouhieddine TH, Tahri S, Ghobrial IM. Monoclonal Gammopathy of Undetermined Significance (MGUS)-Not So Asymptomatic after All. Cancers (Basel). 2020;12(6).

79. Giallongo C, Tibullo D, Parrinello NL, et al. Granulocyte-like myeloid derived suppressor cells (G-MDSC) are increased in multiple myeloma and are driven by dysfunctional mesenchymal stem cells (MSC). Oncotarget. 2016;7(52):85764-85775.

80. Palumbo GA, Parrinello NL, Giallongo C, et al. Monocytic Myeloid Derived Suppressor Cells in Hematological Malignancies. Int $\mathrm{J}$ Mol Sci. 2019;20(21).

81. Romano A, Parrinello NL, La Cava P, et al. PMN-MDSC and arginase are increased in myeloma and may contribute to resistance to therapy. Expert Rev Mol Diagn. 2018;18(7):675-683.

82. Suen $\mathrm{H}$, Brown $\mathrm{R}$, Yang $\mathrm{S}$, et al. Multiple myeloma causes clonal T-cell immunosenescence: identification of potential novel targets for promoting tumour immunity and implications for checkpoint blockade. Leukemia. 2016;30(8):1716-1724.

83. Giallongo C, Tibullo D, Camiolo G, et al. TLR4 signaling drives mesenchymal stromal cells commitment to promote tumor microenvironment transformation in multiple myeloma. Cell Death Dis. 2019;10(10):704.

84. Giallongo C, Tibullo D, Puglisi F, et al. Inhibition of TLR4 Signaling Affects Mitochondrial Fitness and Overcomes Bortezomib Resistance in Myeloma Plasma Cells. Cancers (Basel). 2020;12(8)

85. Kristinsson SY, Tang M, Pfeiffer RM, et al. Monoclonal gammopathy of undetermined significance and risk of infections: a population-based study. Haematologica. 2012;97(6):854-858.

86. Tete SM, Kipling D, Westra J, et al. Monoclonal paraprotein influences 
baseline B-cell repertoire diversity and perturbates influenza vaccinationinduced B-cell response. Exp Hematol. 2015;43(6):439-447.e431.

87. Anderson D, Ali K, Blanchette V, et al. Guidelines on the use of intravenous immune globulin for hematologic conditions. Transfus Med Rev. 2007;21(2 Suppl 1):S9-56.

88. Girmenia C, Cavo M, Offidani M, et al. Management of infectious complications in multiple myeloma patients: Expert panel consensus-based recommendations. Blood Rev. 2019;34:84-94.

89. Vacca A, Melaccio A, Sportelli A, Solimando AG, Dammacco F, Ria R. Subcutaneous immunoglobulins in patients with multiple myeloma and secondary hypogammaglobulinemia: a randomized trial. Clin Immunol. 2018;191:110-115.

90. Haefliger S, Juskevicius D, Höller S, Buser U, Dirnhofer S, Tzankov A. How to resolve a clinical and molecular puzzle: concomitant monoclonal gammopathy of undetermined significance (MGUS) with neutrophilia and clonal hematopoiesis of indeterminate potential (CHIP). Ann Hematol. 2019;98(10):2431-2432.

91. Cohen AL, Sarid R. The relationship between monoclonal gammopathy of undetermined significance and venous thromboembolic disease. Thromb Res. 2010;125(3):216-219.

92. Sallah S, Husain A, Wan J, Vos P, Nguyen NP. The risk of venous thromboembolic disease in patients with monoclonal gammopathy of undetermined significance. Ann Oncol. 2004;15(10):1490-1494.

93. Srkalovic G, Cameron MG, Rybicki L, Deitcher SR, Kattke-Marchant K, Hussein MA. Monoclonal gammopathy of undetermined significance and multiple myeloma are associated with an increased incidence of venothromboembolic disease. Cancer. 2004;101(3):558-566.

94. Rajkumar SV. Thalidomide in newly diagnosed multiple myeloma and overview of experience in smoldering/indolent disease. Semin Hematol. 2003;40(4 Suppl 4):17-22.

95. Detweiler-Short K, Hayman S, Gertz
MA, et al. Long-term results of singleagent thalidomide as initial therapy for asymptomatic (smoldering or indolent) myeloma. Am J Hematol. 2010;85(10):737-740.

96. Rajkumar SV, Dispenzieri A, Fonseca $\mathrm{R}$, et al. Thalidomide for previously untreated indolent or smoldering multiple myeloma. Leukemia. 2001;15(8):1274-1276.

97. Barlogie B, van Rhee F, Shaughnessy JD, Jr., et al. Seven-year median time to progression with thalidomide for smoldering myeloma: partial response identifies subset requiring earlier salvage therapy for symptomatic disease. Blood. 2008;112(8):31223125 .

98. Mateos MV, Hernández MT, Giraldo P, et al. Lenalidomide plus dexamethasone for high-risk smoldering multiple myeloma. N Engl J Med. 2013;369(5):438-447.

99. Mateos MV. When to initiate treatment in smoldering multiple myeloma. Clin Adv Hematol Oncol. 2017;15(10):751753.

100. Paiva B, Mateos MV, Sanchez-Abarca LI, et al. Immune status of high-risk smoldering multiple myeloma patients and its therapeutic modulation under LenDex: a longitudinal analysis. Blood. 2016;127(9):1151-1162.

101. Lonial S, Jacobus S, Fonseca R, et al. Randomized Trial of Lenalidomide Versus Observation in Smoldering Multiple Myeloma. J Clin Oncol. 2020;38(11):1126-1137.

102. Jagannath S, Laubach J, Wong E, et al. Elotuzumab monotherapy in patients with smouldering multiple myeloma: a phase 2 study. $\mathrm{Br} \mathrm{J}$ Haematol. 2018;182(4):495-503.

103. Landgren CO, Chari A, Cohen YC, et al. Daratumumab monotherapy for patients with intermediate-risk or highrisk smoldering multiple myeloma: a randomized, open-label, multicenter, phase 2 study (CENTAURUS). Leukemia. 2020;34(7):1840-1852.

104. Brighton TA, Khot A, Harrison SJ, et al. Randomized, Double-Blind, PlaceboControlled, Multicenter Study of Siltuximab in High-Risk Smoldering Multiple Myeloma. Clin Cancer Res. 2019;25(13):3772-3775.
105. Manasanch EE, Han G, Mathur R, et al. A pilot study of pembrolizumab in smoldering myeloma: report of the clinical, immune, and genomic analysis. Blood Adv. 2019;3(15):2400-2408.

106. Musto P, La Rocca F. Monoclonal antibodies in newly diagnosed and smoldering multiple myeloma: an updated review of current clinical evidence. Expert Rev Hematol. 2020;13(5):501-517.

107. Korde N, Carlsten M, Lee MJ, et al. A phase II trial of pan-KIR2D blockade with IPH2101 in smoldering multiple myeloma. Haematologica. 2014;99(6):e81-83.

108. Wichert S, Juliusson G, Johansson $\AA$, et al. A single-arm, open-label, phase 2 clinical trial evaluating disease response following treatment with BI-505, a human anti-intercellular adhesion molecule-1 monoclonal antibody, in patients with smoldering multiple myeloma. PLoS One. 2017;12(2):e0171205.

109. Golombick T, Diamond TH, Manoharan A, Ramakrishna R. Addition of Rice Bran Arabinoxylan to Curcumin Therapy May Be of Benefit to Patients With Early-Stage B-Cell Lymphoid Malignancies (Monoclonal Gammopathy of Undetermined Significance, Smoldering Multiple Myeloma, or Stage 0/1 Chronic Lymphocytic Leukemia): A Preliminary Clinical Study. Integr Cancer Ther. 2016;15(2):183-189.

110. Golombick T, Diamond TH, Manoharan A, Ramakrishna R. Monoclonal gammopathy of undetermined significance, smoldering multiple myeloma, and curcumin: a randomized, double-blind placebocontrolled cross-over $4 \mathrm{~g}$ study and an open-label $8 \mathrm{~g}$ extension study. Am J Hematol. 2012;87(5):455-460.

111. Korde N, Roschewski M, Zingone A, et al. Treatment With CarfilzomibLenalidomide-Dexamethasone With Lenalidomide Extension in Patients With Smoldering or Newly Diagnosed Multiple Myeloma. JAMA Oncol. 2015;1(6):746-754. 